\title{
Novel Sub-Harmonic Injection-Locked Balanced Oscillator
}

\author{
N. Siripon ${ }^{1}$, M. Chongcheawchamnan, and I. D. Robertson \\ Microwave and Systems Research Group (MSRG), School of Electronics, Computing and Mathematics, \\ University of Surrey, Guildford, Surrey, GU2 7XH, United Kingdom. \\ 1eep1ns@ee.surrey.ac.uk
}

\begin{abstract}
A novel sub-harmonic injection-locked balanced oscillator is proposed. The circuit provides two outputs with a $180^{\circ}$ phase difference by employing a transmission line section for impedance transformation to meet the oscillation conditions. A coupling network is connected at the mid-point of the transmission line to inject the sub-harmonic frequency. This eliminates the need for a circulator or balun. The circuit is small and consumes low DC power. Under the locking state, the circuit provides double the injection frequency and also the phase noise of the two outputs is substantially improved.
\end{abstract}

\section{INTRODUCTION}

Local-oscillator phase noise is a key performance parameter in a communication system, since, for example, it affects the rejection of adjacent channel interference (ACI) and the ability to detect weak signals. Therefore, low phase noise oscillators are needed for the next generation of millimeter wave communication. A technique popularly used to stabilize the free-running frequency is the injectionlocked oscillator. This also introduces an improvement to the phase noise performance of the oscillator. The prospect of using the synchronous oscillator to lock a signal at the same frequency (the free-running frequency) has been studied widely in the literature [1]-[2]. A sub-harmonic injection-locked technique has been also proposed as a particular technique for optical synchronization of the remoted local oscillator at microwave and millimeter-wave applications [3]-[4]. Recently, an injection-locked push-pull or balanced oscillator was proposed and applied to a spatial power combining array antenna [5]. Though the structure proposed in [6] can be applied with an external subharmonic injection signal, a large circuit area due to the use of a transmission line to achieve $180^{\circ}$ phase difference outputs is its main disadvantage.

In this paper, we propose a novel structure for a subharmonic injection-locked balanced oscillator (SILBO). The balanced oscillator designed approach is based on the extended resonance technique [7], which is described in section II. Section III shows the measurement results. The stabilized injection-locked oscillating signal and the locking range with respect to the variety of the injection power levels are investigated. It also shows the balanced amplitude and phase difference between the two output of this subharmonic injection-locked oscillator under the locking state by using the external mixers. This experiment was set up in order to ensure the amplitude and phase output properties of the balanced oscillator. The circuit gives many advantages, including a simple design technique and low DC power consumption. In addition, the circuit size is relatively small because a circulator and balun at the injection port and input/output ports, respectively, are eliminated.

\section{DESIGN TECHNIQUE}

The proposed circuit diagram is shown in Fig. 1. The sub-harmonic injection-locked balanced oscillator is designed and constructed on FR4 and uses Siemens CFY30 GaAs FET devices. The oscillation condition is met using a two-terminal negative resistance method [8]. In this case a short open circuit stub is used as a series feedback to provide the negative resistance. The use of a transmission line between the two active devices is used to satisfy the oscillation conditions and the $180^{\circ}$ phase difference between the oscillating output signals [9]. Then, the matching network $\left(\mathrm{M}_{1}\right)$ is required to fulfill the oscillator condition at the design frequency. This causes the input impedance of one active device to be the load impedance for the second active device seen at the gate of the second device, and vice versa. Its circuit equation in the free-running state is given by

$$
Z_{I N C X}(V, \omega)+Z_{D X}(V, \omega)=0
$$

where $Z_{D X}(V, \omega)$ is the input impedance, $Z_{N C}(V, \omega)$ is the load impedance and $X$ is 1 or 2 . The impedance of each device, $Z_{D 1}(V, \omega)$ and $Z_{D 2}(V, \omega)$, is $R_{L}-R-j X$. By using a transmission line, the impedance of the first device, $Z_{D 1}(V, \omega)$, is transformed such that the impedance $Z_{N C}(V, \omega)$, seen by this active device is $R_{L}+R+j X$. Then the two devices resonate with each other.

The synchronized sub-harmonic signal is injected to the balanced oscillator through the matching network $\left(\mathrm{M}_{2}\right)$ at sub-harmonic frequency, free-running frequency/2. Instead of using the 
circulator, the matching network is connected at the mid-point of the transmission line so that the symmetrical structure is still maintained. Thus, the advantage of this topology is that a circulator is not required and the circuit also provides a perfect LO isolation at the injection port.

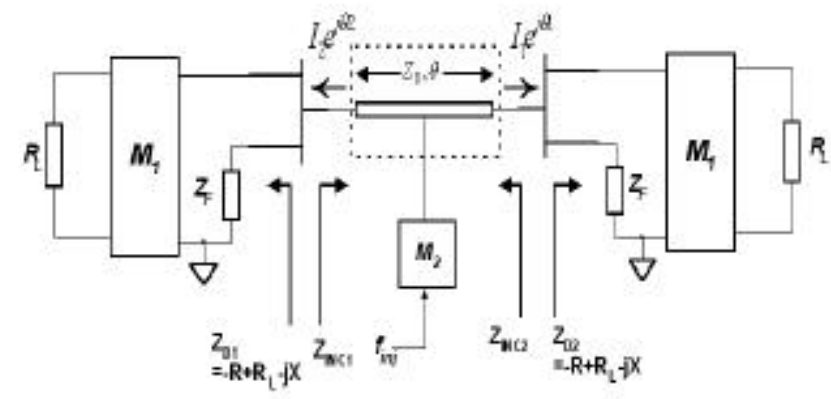

Fig. 1: The proposed sub-harmonic injection-locked balanced oscillator diagram

Fig. 2 shows the layout of the sub-harmonic injectionlocked balanced oscillator. For the injection matching network $\left(\mathrm{M}_{2}\right)$ shown in Fig. 2., a coupled line section is used to couple the sub-harmonic signal. This coupled line functions as a bandpass filter, which is open circuited at the oscillating frequency but passes the sub-harmonic frequency. Therefore, good return loss is obtained at this injection port. It should be noted that the DC is also isolated by using this structure.

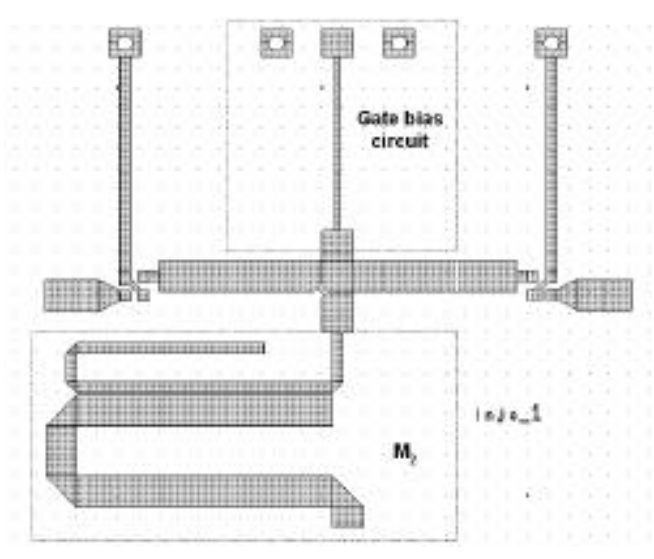

Fig. 2: The Layout of the sub-harmonic injection-locked balanced oscillator circuit

\section{MEASUREMENT RESULTS}

The biasing is provided through a tee junction at each output port. The circuit was biased at $\mathrm{V}_{\mathrm{DS}}=2.5 \mathrm{~V}$ and $\mathrm{V}_{\mathrm{GS}}=-0.4 \mathrm{~V}$. The free-running frequency of this balanced oscillator was measured by terminating a 50ohm load at the injection port. The oscillator provides a free-running frequency at $1.9539 \mathrm{GHz}$. with a power of $4.5 \mathrm{dBm}$. The circuit consumes $23.27 \mathrm{mWatt}$.
To investigate the sub-harmonic injection-locking, a phase-locked signal generator is connected to the matching network to feed the injection signal into the oscillator. Fig. 3 shows the output spectrum of the subharmonic injection locked balanced oscillator. The external injection frequency was $977.86 \mathrm{MHz}$ with a power level of $-2.5 \mathrm{dBm}$. The sub-harmonic injection locked oscillator provides better oscillating frequency stability and reduces the phase noise.

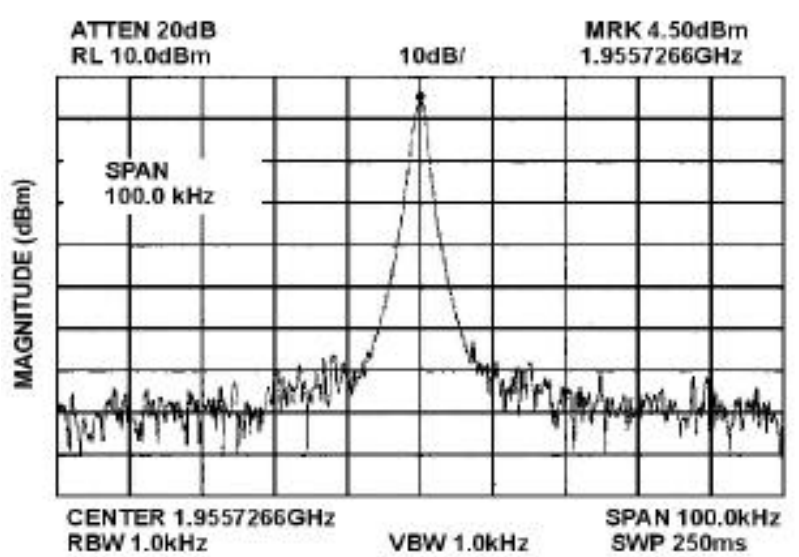

Fig. 3: The measured injection locking frequency signal.

The locking range with respect to the injection power level was then measured. Fig. 4 shows the locking range of this sub-harmonic injection-locked balanced oscillator. The locking ranges of this oscillator are 110 $\mathrm{kHz}$ and $600 \mathrm{kHz}$ with the injection power levels of -5 $\mathrm{dBm}$ and $2.5 \mathrm{dBm}$, respectively.

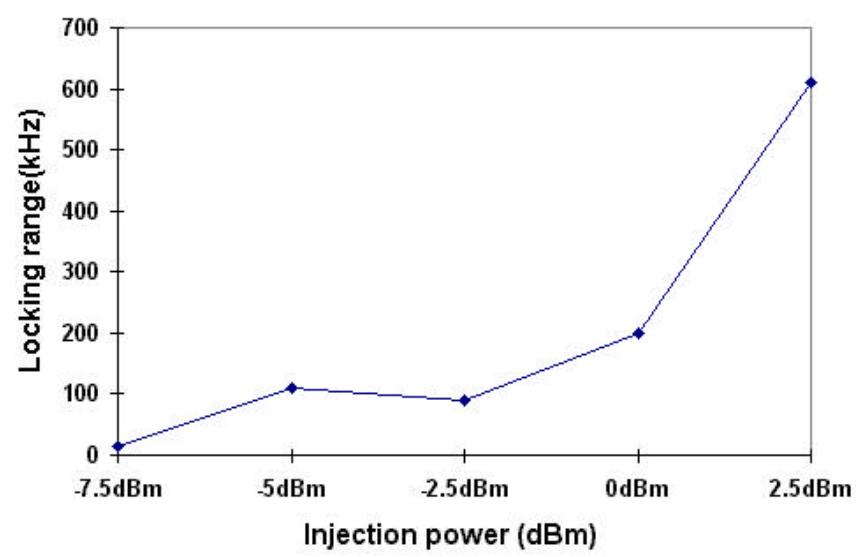

Fig. 4: The measured locking range with respect to the injection power level. 


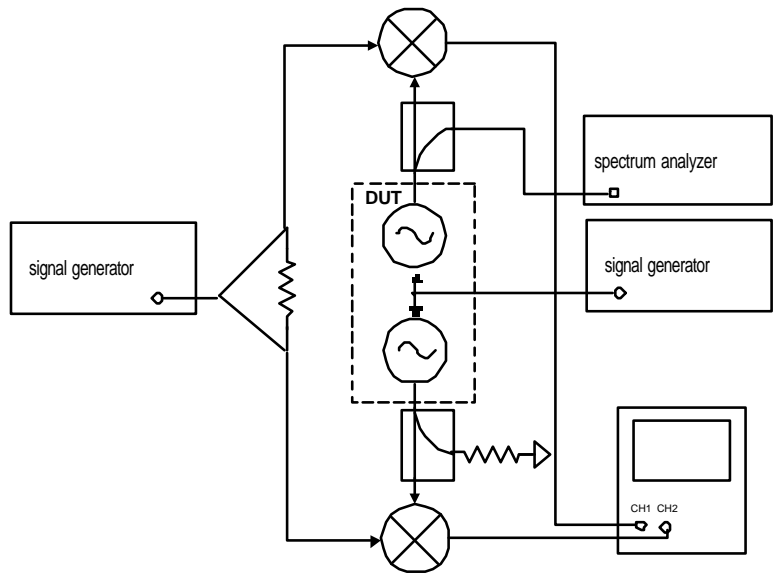

Fig. 5: Phase measurement test bench setup for subharmonic ILBO

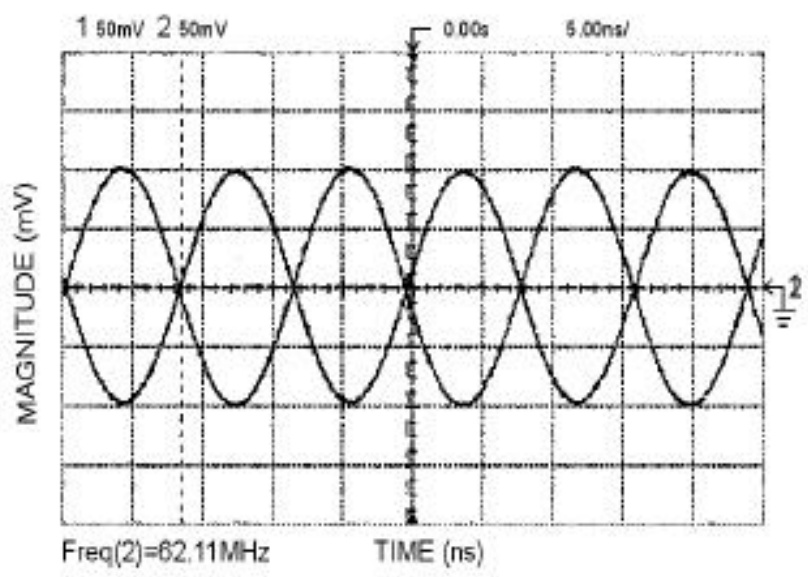

Fig. 6: Measured phase difference between the two outputs of the sub-harmonic ILBO.

In order to investigate the amplitude balance and phase difference between the two outputs of the SILBO, the test bench is setup as shown in Fig. 5. An RF signal is equally split by a Wilkinson divider. Two in-house mixers are used for down-converting the LO signals to IF. Two $21.37 \mathrm{~dB}$ couplers were constructed on FR4. The phase difference between the direct ports of these two couplers is $0.345^{\circ}$. The first coupler is used for monitoring the injection-locked spectrum at the first port while the second one is used for compensating a phase delay. It is noted that the phase delay of these two couplers must be equal.

An external sub-harmonic signal of $981.055 \mathrm{MHz}$ at 0 $\mathrm{dBm}$ was injected into the balanced oscillator which was biased at $2.63 \mathrm{~V}$ drain-source and $-0.9 \mathrm{~V}$ gatesource bias. A $0 \mathrm{dBm} 1.9 \mathrm{GHz} \mathrm{RF}$ signal is applied to the Wilkinson divider input. Under the locking state, the circuit provides a locked signal of $1.96211 \mathrm{GHz}$, which is twice the frequency of the injection signal and close to the fundamental frequency signal of the oscillator. Fig. 6 shows the $62.11 \mathrm{MHz}$-IF signals from the two mixers, after the RF signal is down-converted, monitoring the phase difference due to the subharmonic injection-locked oscillator outputs. Thus, it is clearly shown that the balanced oscillating signals still maintain $180^{\circ}$ phase difference under locking conditions.

\section{CONCLUSIONS}

The sub-harmonic injection-locked balanced oscillator has been presented. The oscillator provides a pair of out-of-phase outputs. By employing the symmetrical configuration, the properly coupled transmission line is used as the matching network at the centre of the circuit. The external locking signal, whose frequency is approximately half the free-running frequency, is injected to the center of the transmission-line between the devices. As a result, locked output signals are obtained. Under the locking state, the oscillator provides double the injection frequency with a significant phase noise improvement, whilst still maintaining $180^{\circ}$ phase difference as shown in the experiment section. The advantage of this circuit is that it is simple to design since the design eliminates the need of the circulator for the injection signal. The $\mathrm{LO} / \mathrm{RF}$ balun is also not needed in real applications such as balanced mixers. This reduces the circuit size and also minimises cost for MMIC fabrication. It is found that the SIBLO consumes low DC power. Furthermore, this technique can improve the phase noise in the balanced oscillator, which can cause many difficulties in the communication applications.

\section{ACKNOWLEDGMENTS}

The authors wish to acknowledge to the Thai government and Mahanakhorn University of Technology for their financial support. The authors are also grateful to the Electronics Workshop staff of the University of Surrey for their technical support.

\section{REFERENCES}

[1] R. Adler, "A Study of Locking Phenomena in Proceeding of the I.R.E. and Waves and Electrons, pp. 351-357, June 1946.

[2] C.C. Huang and T.H. Chu, "Analysis of MESFET Injection-Locked Oscillators in Fundamental Mode of Operation", IEEE Transactions on Microwave Theory and Techniques, vol. 42, pp.1851-1857, October 1994.

[3] A.S. Daryoush, "Optical synchronization of millimeter wave oscillator for distributed architecture (invited paper), IEEE Transactions on Microwave Theory and Techniques, vol. 38, pp.467-476, May 1990. 
[4] A.S. Daryoush, P.R. Herczfeld, Z. Turski, and P.K. Wahi, "Comparison of Indirect Optical Injection-locking Technique of Multiple X-band Oscillators," IEEE Trans. Microwave Theory and Techniques, MTT-34, No 12, pp. 13631370, December 1986.

[5] A. Mortazawi, and B.C. De Loach, "Multiple element oscillators utilising a new power combining technique", IEEE Int. Microwave Symp. Dig., pp. 1093-1096, 1992.

[6] G. Gonzalez and O.J. Sosa, "On the Design of a Series-Feedback Network in a Transistor Negative-Resistance Oscillator', IEEE Transactions on Microwave Theory and Techniques, vol. 47, No. 1, pp.42-47, January 1999.

[7] K.S. Ang, S. Nam, and I.D. Robertson, "A Balanced Monolithic Oscillator at K-Band", IEEE Radio Frequency Integrated Circuits (RFIC) Symposium, pp.163, 1999. 